\title{
Atherosclerosis: what is it and why does it occur?
}

\author{
M J Davies, $\mathrm{N}$ Woolf
}

The limits of what is encompassed by the term atherosclerosis are fundamental to understanding the pathogenesis of the disease and the ways in which clinical symptoms are produced.

\section{Basic definitions of atherosclerosis}

Atherosclerosis is an intimal disease of arteries within a range of size from the aorta down to approximately $3 \mathrm{~mm}$ external diameter. The distribution of the disease is far from uniform; some arteries such as the internal mammary are largely spared while others such as the coronary arteries are at high risk. Similarly, in the same individual the degree of coronary, cerebral, and carotid atherosclerosis is often disparate.

Atherosclerosis is a focal not a diffuse intimal disease. The focal nature can be appreciated by examining the intimal surface of an opened artery, when discrete lesions or plaques are visible.

Lipid and the connective tissue matrix proteins are the two major components of plaques. The lipid may be extracellular or intracellular and both forms are usually found in advanced plaques. Most lipid-containing foam cells are macrophages derived originally from circulating monocytes that have entered the intima. Connective tissue matrix proteins are synthesised by smooth muscle cells. The matrix protein is collagen. This is present in large amounts, conferring mechanical strength to the tissues and thereby holding the plaque together as a stable structure.

Some of the processes involved in atherosclerosis do occur in other vascular diseases, and it is important that the definition of atherosclerosis is not blurred or confused. The medial smooth muscle hypertrophy and subsequent fibrosis that occur in hypertension and affect smaller arteries are not atherosclerosis. The medial calcification that occurs with age in the lower limb arteries is not atherosclerosis. The use of the generic term "arteriosclerosis", often converted into the lay term "hardening of the arteries", leads to tremendous confusion by lumping all of these entities into one age-related arterial disease.

Isolated intimal smooth muscle proliferation is not atherosclerosis. Smooth muscle proliferation is a general response to injury by the arterial wall and does occur in atherosclerosis. But it is neither unique to nor specific for atherosclerosis. Many animal models of arterial disease have been created in which vascular damage is induced by intra- luminal balloon injury or external compression. A localised intimal smooth muscle proliferation occurs which simulates one component of the human atherosclerotic plaque. Post angioplasty stenosis in humans is an example of such a response to injury by an atherosclerotic vessel but the lesion created is different from a human plaque.

\section{Clinical symptoms and atherosclerosis}

Plaque formation begins in early life, by the third decade advanced plaques are almost ubiquitous in Western populations. Therefore there is a long presymptomatic phase before the disease appears above the clinical horizon.

The conversion from a person with atherosclerosis, but no clinical symptoms, to a patient with symptoms is an important breakpoint and may be largely determined by complications most of which involve thrombosis developing in association with plaques. Any analysis of therapy should consider separately the prevention of plaque formation and the reduction of plaque complications.

Clinical symptoms depend on four mechanisms. The primary process of atherogenesis (that is, lipid accumulation and connective tissue matrix production by smooth muscle cells) may increase the volume of one or more key plaques so that they encroach on the lumen and become flow limiting. Second, a plaque may enter an unstable phase and be complicated by the formation of a thrombus. This thrombus may itself encroach on, or occlude, the arterial lumen, or break away embolise and impact in smaller more distal vessels. Third, though atherosclerosis is a focal disease it is associated with a generalised abnormality of vascular tone in affected arteries that favours vasoconstriction often at inappropriate times such as on exercise. Finally, medial atrophy and destruction secondary to intimal atherosclerosis may lead to aneurysm formation. Many patients suffer combinations of all these mechanisms.

\section{Pathogenesis of atherosclerosis}

Any consideration of what comprises human atherosclerosis raises the following salient issues:

(a) What is the origin of the intimal lipid?

(b) Why is there an intimal inflammatory response in which monocytes migrate into and become permanently resident in the intima? 
Figure 1 Diagram to show plasma low density lipoprotein (LDL) entering the subendothelial space and being oxidised, and the consequent formation of a foam cell.

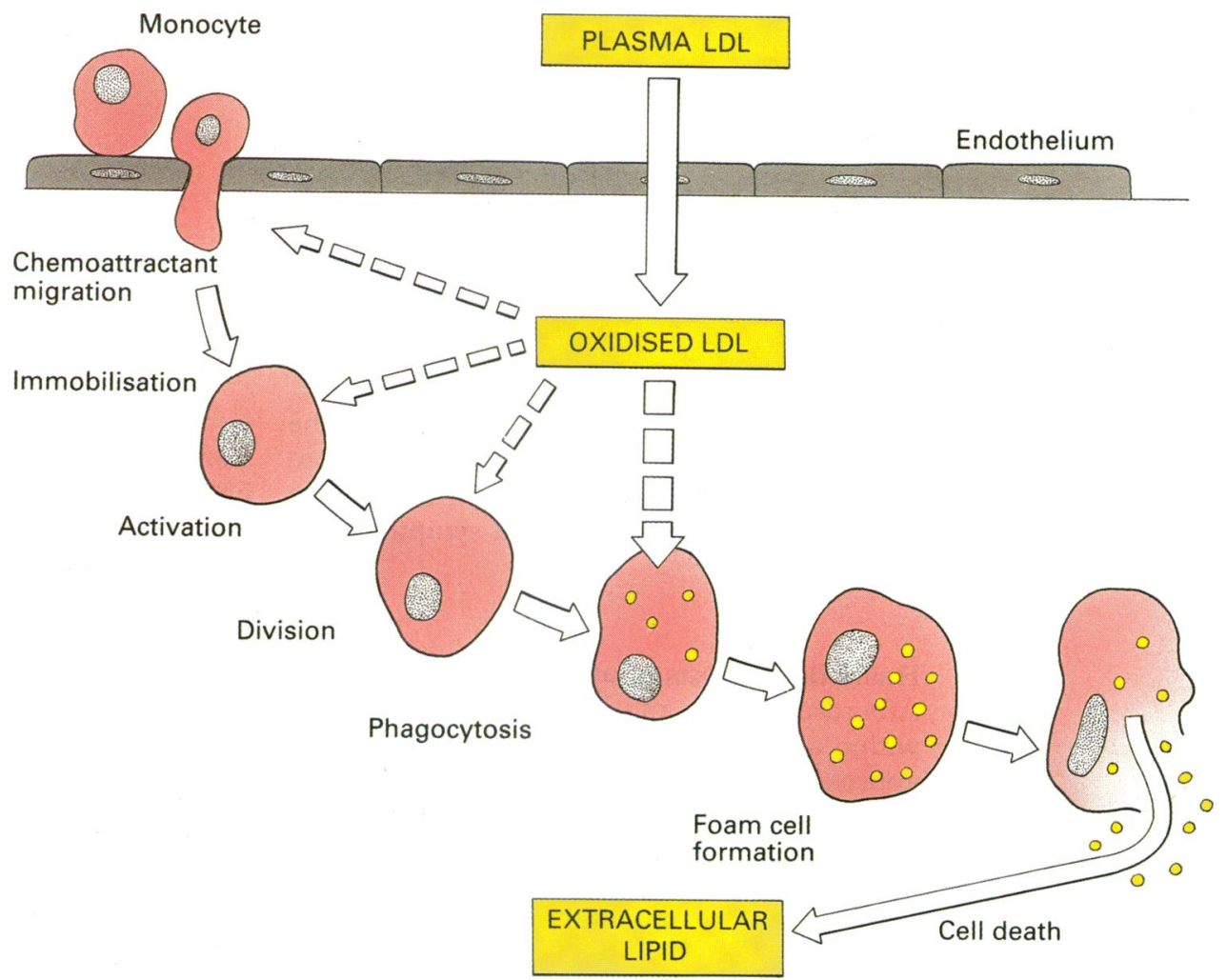

(c) How is smooth muscle proliferation and collagen synthesis within the intima initiated and maintained?

(d) What processes invoke thrombosis over unstable plaques?

Only when the answers to these questions, all of which are discussed in this supplement, are known can truly rational therapy to control the progression of atherosclerosis be initiated.

In the broadest terms atherosclerosis is now recognised to be an immune/inflammatory response of the intima to injury. ${ }^{1}$ It is also being increasingly realised that the injury is initiated by lipid. There is, however, a paradox in that native plasma lipids, in particular low density lipoproteins, though they freely enter the intima, do not initiate an inflammatory response, are not ingested by monocytes, and do not damage tissue. Oxidation or alteration of low density lipoprotein will however radically alter this passive state (fig 1 ): oxidised lipid is chemotactic for monocytes, induces migration, initiates inflammatory $\vec{\bullet}$ responses, is toxic to monocytes, and is avidly $\oplus^{\circ}$ ingested by them via scavenger receptors. ${ }^{2}$ Lipid uptake via this receptor is not down regulated by intracellular lipid and the monocyte becomes stuffed with lipid to become a "foam cell".

Smooth muscle proliferation is controlled $\stackrel{2}{\Rightarrow}$ by growth factors released from a wide range $\frac{3}{3}$ of cells (fig 2) including platelets, endothelial cells, macrophages, and other smooth muscleọ cells. Inflammatory reactions readily induce growth factor production. All of these processes are considered in individual chap-? ters of this supplement.

Any consideration of human atherosclerosis cannot deny the role of lipid in intimal injury. This lipid is largely plasma derivedo and results from plasma concentrations that are greater than ideal for that individual. It is important to remember that the plasma lipid $\bar{N}$ profile of an individual is the result of interac-o tions between genetic and environmental fac- $N$
Figure 2 Influence of growth factor production on smooth muscle proliferation.

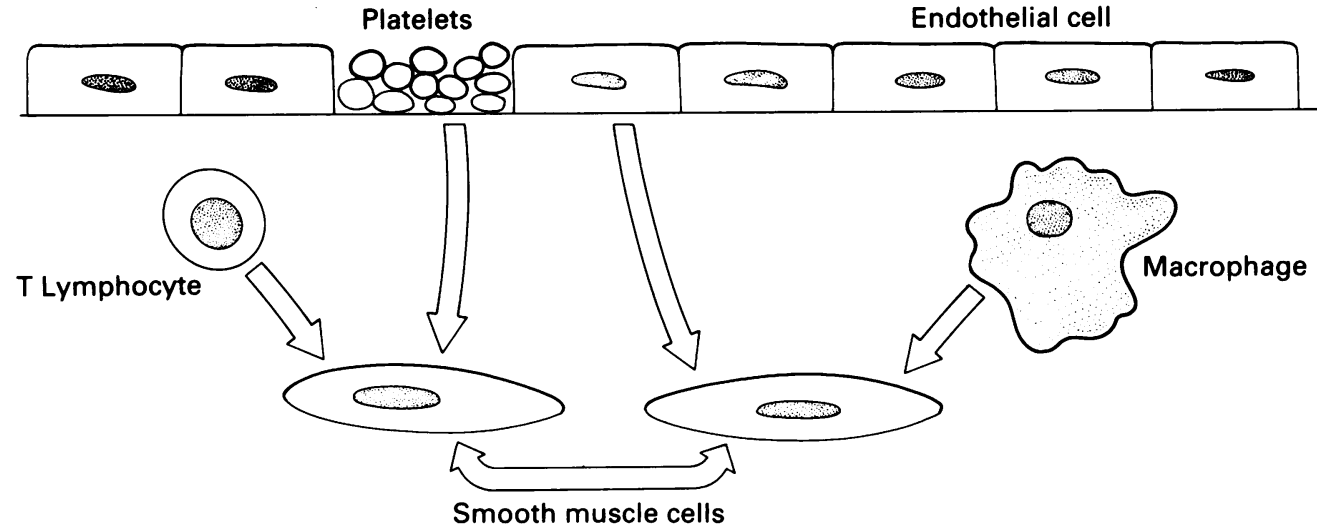




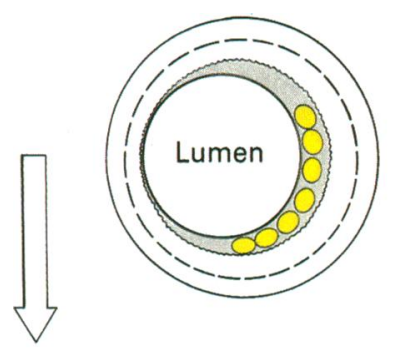

\author{
FATTY STREAK \\ containing: \\ Foam cells $\bigcirc$ \\ Intracellular lipid
}

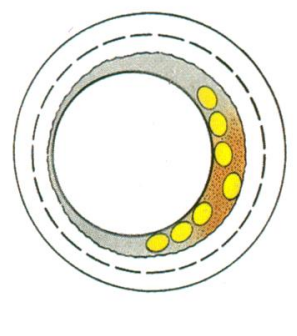

TRANSITIONAL PLAQUE containing:

Foam cells $\bigcirc$

Intracellular lipid

Extracellular lipid

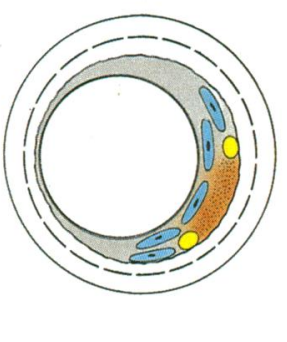

\section{ADVANCED FIBROLIPID PLAQUE containing:}

Extracellular lipid core

Smooth muscle cells $\infty$

Foam cells $\bigcirc$

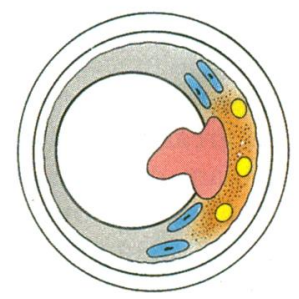

COMPLICATED PLAQUE WITH THROMBUS FORMATION

Figure 3 Human plaque progression. The fatty streak contains only foam cells. Extracellular lipid, smooth muscle cells, and collagen appear successively and lead to an advanced plaque on which the complication of thrombosis can arise.

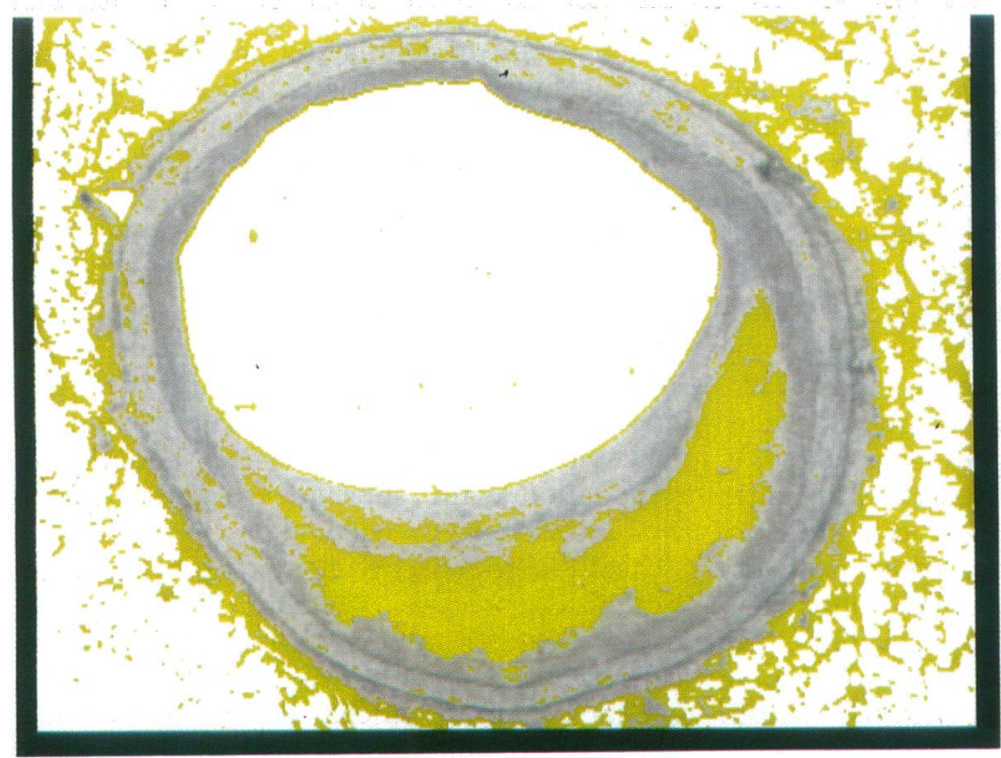

Figure 4 Micrograph showing cross section of human coronary plaque showing a large pool of lipid (coded yellow) encapsulated in the plaque. tors the most important of the latter being diet.

I

\section{Progression of plaques in humans}

An inherent drawback of any human necropsy study is that the state of the artery is seen at one moment only. Nevertheless, there are numerous morphological and histological descriptions of the different types of human plaque with speculation about how each is related temporally. In reality the only safe conclusion is that large plaques must evolve from smaller plaques but over what period of time is unknown. The studies by Stary have provided many answers about plaque evolution. ${ }^{3}$ Detailed studies were made of the arteries in young subjects, from infancy to over 30 years of age, dying suddenly from non-cardiac disease. The cases were divided into cohorts by age. It was therefore possible to see the age at which lesions of any particular type first appeared, and the sequence of plaque progression could be inferred.

The first observation was that in all human infants focal thickening of the intima develops due to smooth muscle proliferation. Stary has argued cogently that this is a ubiquitous adaptive response, and not early atherosclerosis. Nevertheless, it does mean that the human coronary intima already contains smooth muscle cells, and is presensitised to the later development of atherosclerosis if certain conditions prevail.

The first atherosclerotic lesions which appear are fatty dots or streaks barely raised above the intimal surface. Each lesion is made up by a focal collection of lipid-filled foam cells within the intima. On one hand there is evidence that such simple fatty streaks or dots are the precursor of larger plaques. Pathological studies show that transitional forms exist between fatty streaks and advanced plaques, and that advanced plaques develop in the sites where fatty streaks are most frequent. On the other hand it is clear that not all fatty streaks progress. Necropsy surveys of infants from geographical populations in which the advanced atherosclerosis does not develop and there is a negligible incidence of ischaemic heart disease show large numbers of fatty streaks. ${ }^{4}$

The cohort studies by Stary show that progression beyond the fatty streak stage is associated with a sequence of changes starting with the appearance of extracellular lipid which begins to form a core to a lesion that is becoming more elevated (fig 3). Smooth muscle cells migrate into and proliferate within the plaque, forming a layer over the luminal side of the lipid core. More and more collagen is produced and plaque size increases. The process culminates in what is known as a raised fibrolipid or advanced plaque. In the aorta such plaques may be a centimetre or more in length.

The aorta and coronary arteries of adults show all stages of plaque development, implying that new lesions are generated throughout 
adult life. The work by Stary indicates that a fully developed plaque can take up to $10-15$ years to develop.

Raised fibrolipid plaques have a very characteristic microanatomy (fig 4 ) with a core of extracellular lipid separated from the media by smooth muscle cells and covered and separated from the lumen by a thick cap of collagen-rich fibrous tissue containing smooth muscle cells. Surrounding the lipid core are lipid-filled foam cells. Much of the core lipid is thought to be derived from the death of lipid-containing macrophage foam cells and the release of their intracytoplasmic content (fig 1). This process of plaque necrosis is an important facet of human disease and has considerable influence on the later development of thrombosis. Even in the same individual there are major plaque to plaque variations in the relative proportions of extracellular lipid and collagen that are present.

The raised fibrolipid or advanced atherosclerotic plaque is the substrate on which plaque complications develop, and therefore clinical symptoms appear. The evidence for this view comes from large geographical studies of the number of raised plaques at necropsy in the aortas and coronary arteries in subjects who did not die from ischaemic heart disease. In any specific geographical population the mean number of plaques present mirrors the frequency of death from ischaemic heart disease in the same population. ${ }^{5} \mathrm{~A}$ further fact to emerge is that individuals with hypertension have more aortic and coronary plaques than normotensive individuals. ${ }^{67}$ Whereas the number of advanced plaques is an important determinant of the risk of developing clinical symptoms there will always be unlucky individuals who have very few plaques but develop acute infarction or die suddenly because of thrombosis on a single strategically placed plaque, for example at the origin of the left anterior descending coronary artery.

Though the term "advanced", adopted by Stary, is accurate in indicating the lesion has developed over years it may be misleading in a clinical setting. Most advanced plaques are not angiographically visible. This unfortunate fact means that accurate angiographic assessment of the amount of wall disease, as distinct from the degree of stenosis, is impossible. The angiogram can indicate focal narrowing of the lumen, nothing more.

The reasons for the angiographic insensitivity in the detection of plaques lie in the medial changes that develop in atherosclerotic vessels. When intimal plaques develop the arterial media undergoes a remodelling process which increases the external diameter of the vessel. The plaques are therefore accommodated without any reduction in the cross sectional area of the lumen. ${ }^{8}$ Only when this capacity for remodelling is overcome do plaques compromise the lumen and become angiographically visible. A second mechanism is that behind plaques the media atrophies and can virtually vanish, the internal elastic lamina may rupture, and the whole plaque can shift outward. In extreme cases the plaque is almost extruded from the original outline of the vessel.

The result of both these medial changes is that it is impossible ever to be sure that an angiographically normal segment of artery $\frac{T}{\mathbb{D}}$ does not contain a hidden plaque. A corollary is that the degree of lumen stenosis bears no. constant relation to plaque size. Yet another corollary is that the term "new" as applied to a stenosis appearing between two angiograms $\frac{-}{5}$ is a misnomer: the plaque may have been $\overline{\bar{c}}$. there for years and have recently undergone $a \stackrel{\Phi}{\circ}$ change making it angiographically visible.

\section{Animal models of atherosclerosis}

There is one incontrovertible feature of $\overrightarrow{\vec{\omega}}$ animal models. This is that only when plasma $\stackrel{\circ}{\circ}$ lipid concentrations are increased is a disease produced which has morphological similari- 8 ties to atherosclerosis in humans.

Plasma lipid concentrations can be raised 0 either by high fat diets or by the use of animals that have an inherent defect in lipid metabolism leading to hyperlipidaemia.

All animal models have merits and demer- $\frac{\text { O }}{2}$ its. Rabbit models based on the use of high fat diets are cheap and relatively quick. An increase in the percentage of the aortic intima occupied by stainable lipid can be seen $12 \stackrel{2}{2}$ weeks after the start of a high fat diet. This $\vec{\bullet}$ provides ideal circumstances for testing antiatherogenic drugs. But are aortic lesions produced by very unphysiological diets over a short period of time analogous to human atherosclerosis? The great bulk of the lipid in the aorta is within foam cells: in effect what $\frac{}{\Phi}$ has been produced are numerous giant fatty streaks not advanced plaques. Hereditary $\frac{0}{3}$ defects in lipid metabolism such as that found in the Watanabe or St Thomas' rabbit do allow the effect of more chronic, less extreme hyperlipidaemia and do reproduce plaques $\stackrel{\mathbb{D}}{\mathbb{D}}$ with lipid cores. Long-term dietary athero- $\frac{\mathscr{7}}{-}$ sclerosis in both primate and porcine models $\frac{3}{3}$ will also reproduce a more typically human type of plaque but these models are expensive.

The value of animal models is that they have allowed sequential studies of plaque $\frac{D}{0}$ development in terms of cellular events. The animal models are consistent in showing that o the initial event is adhesion of monocytes to $\tilde{O}$ an intact endothelial surface followed by their ${ }_{\mathrm{N}}$ migration into the intima. Endothelialo denudation (that is, exposure of subend-ce othelial matrix) which causes platelet adhesion or activation is not present and therefore? does not play any part in plaque initiation. ${ }^{9} \frac{7}{2}$ Once plaques are established endothelial $\stackrel{\circ}{\mathbb{D}}$

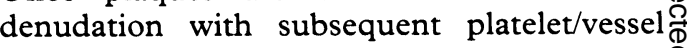
wall interactions do occur. ${ }^{10}$

Lipid ingestion by monocytes within the intima leads to the appearance of foam cellso in increasing numbers. As lipid-filled foamড cells accumulate within the intima defects doo appear in the endothelial surface over which? platelets do adhere. Further cellular events very much follow the sequence described by Stary in human disease. 
Clinical symptoms and atherosclerosis STABLE EXERTIONAL ANGINA

Clinical angiographic studies show that most patients with stable angina have one or more areas of high grade stenosis. Stenoses that reduce the luminal diameter by more than $50 \%$, when compared with adjacent segments judged to be normal, are considered to be flow limiting when myocardial oxygen demand rises.

The angiographic assessment of the degree of stenosis is at best semi-quantitative, and liable to unpredictable errors. ${ }^{11}$ The major source of error lies in the comparison with a reference "normal" segment. This segment may in fact be diffusely narrowed or even dilated. Computerised reconstruction of the normal distal tapering of the vessel will only partly overcome this difficulty. A case can be made for the use of absolute measurements of lumen size in sequential angiograms. Even so the capacity of the coronary artery to remodel itself makes sequential change difficult to interpret.

The morphological characteristics of high grade stenoses have several variables. Plaques may be eccentric (that is, occupy only an arc of the vessel circumference) or be concentric and surround the entire circumference. In eccentric plaques there is a residual arc of vessel in which the medial structure is intact and may be capable of vasomotor tonal variation. In concentric plaques both the splinting effect of the plaque and the associated general loss of media mean that there is no capacity for tonal variation in lumen size.

The reported frequency of stenoses which retain a segment of normal media varies widely in pathological studies. ${ }^{12} 13$ This variation reflects several factors. Plaques that are not yet angiographically visible are far more likely to be eccentric than plaques that are already causing stenosis. The frequency of eccentric lesions falls as the degree of stenosis rises. The situation can, however, be summed up in the fact that most patients with stable angina will have at least one stenosis of more than $50 \%$ by diameter which has the potential to vary the degree of obstruction as vasomotor tone changes.

A further variable in plaques causing stenosis is the relative proportion of collagen and lipid. At one extreme the plaque may be virtually all collagen: at the other extreme $70 \%$ of the plaque volume may be lipid. The proportion of lipid tends to fall with increasing stenosis but there are many exceptions to this rule. Overall, however, there is a marked trend for high grade lesions to be predominantly collagenous, suggesting that smooth muscle proliferation, however initiated, is the factor leading to the later stages of obstruction. High grade lesions ( $>70 \%$ by diameter) are often histologically complex with several layers of collagen of different structure: distal and proximal to the main plaque concentric intimal smooth muscle proliferation occurs. A high proportion of patients $(>75 \%)$ with stable angina, irrespective of whether they have had previous myocardial infarction, have lesions in which the lumen at the point of maximum stenosis has several channels. ${ }^{12}$ These appearances result from the organisation of an occluding thrombus.

The pathological basis of most cases of stable angina is therefore the stable plaque causing high grade stenosis. "Stable", as applied to a plaque, means that no acute thrombosis is occurring.

\section{Endothelial status over stable plaques}

Use of the term stable with regard to a plaque does not imply that no inflammatory activity or endothelial damage is occurring. Animal models show that once advanced plaques are established endothelial denudation injury and localised adhesion of platelets are common. Human studies in which coronary arteries were perfused with gluteraldehyde and the endothelial surface examined by scanning electron microscopy confirm that similar changes occur. ${ }^{1415}$

The endothelial surface over plaques shows loss of individual cells, or several contiguous cells, exposing the subendothelial matrixover which a monolayer of platelets forms (fig 5). Adjacent to these areas of endothelial loss macrophage foam cells are present. The presence of these foci of denudation over human coronary plaques is the rule rather than the exception. These findings imply that the endothelium over atherosclerotic lesions is undergoing constant regeneration and that many individual endothelial cells are immature. These microthrombi consisting of a monolayer of platelets do not mechanically obstruct flow and fall far short of being angiographically visible. These platelets, however, have the potential to stimulate smooth muscle proliferation within the plaque. The lesions also show that plaque stability is a relative term and that some element of microthrombosis is an integral part of the progression of advanced lesions. Endothelial denudation injury is associated with foam cell infiltration in the superficial layers of the plaque and is a marker of the inflammatory activity in the plaque.

\section{Unstable angina and acute myocardial infarction}

Unstable angina and acute myocardial infarction, though often regarded as clinically distinct, form the two ends of a spectrum which results from thrombosis occurring over unstable plaques. The difference between the two conditions lies in the effect these thrombi have on the myocardium. ${ }^{16}$

Thrombosis of sufficient magnitude to obstruct flow and be visible angiographically is due either to superficial or deep injury to the plaque. ${ }^{17}$

Superficial injury is the continuation of the process of focal endothelial denudation that occurs over many plaques. When large confluent areas of denudation occur, thrombi build up in size and may even become occlusive. Plaques with this degree of endothelial 


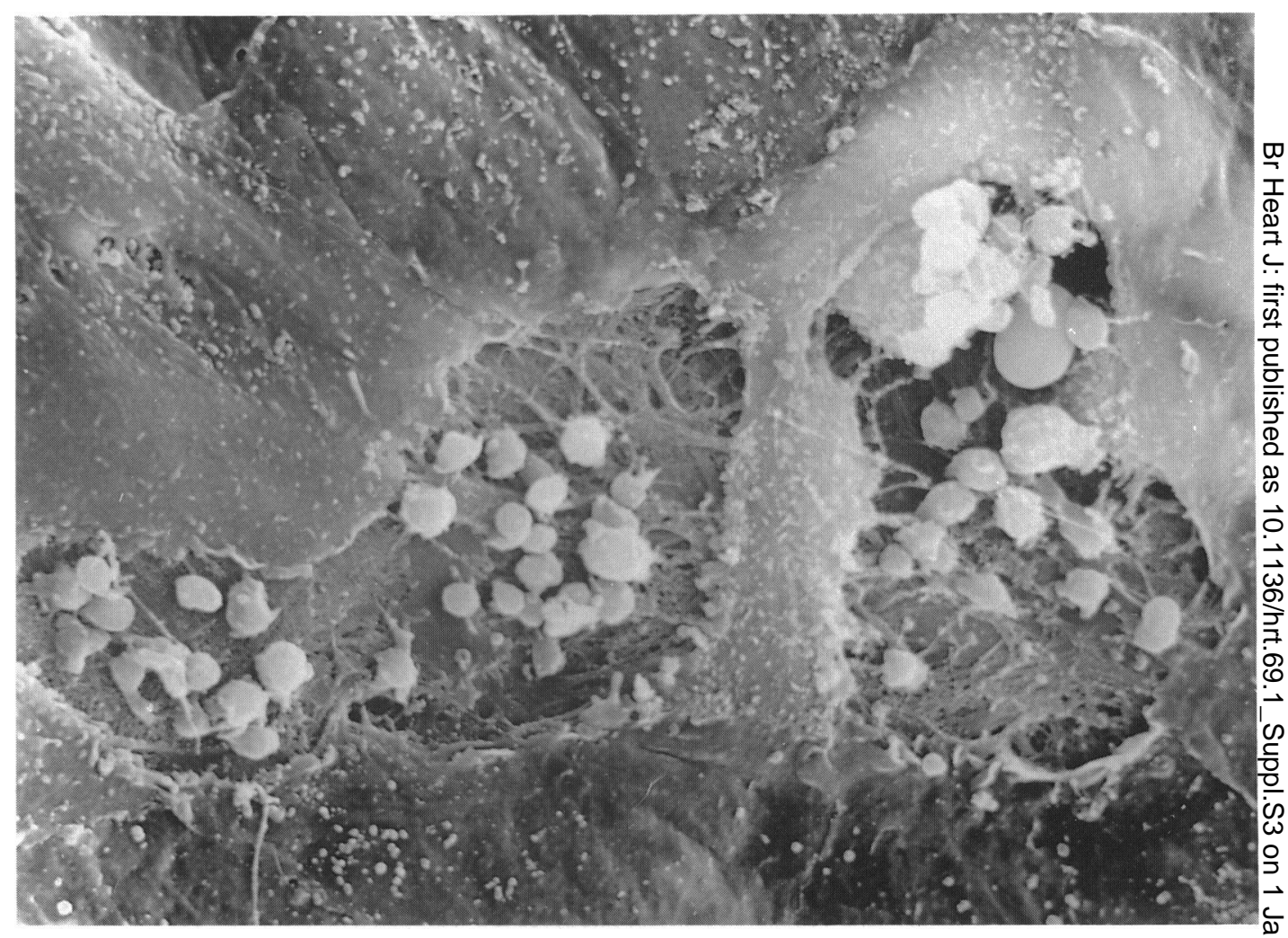

Figure 5 Electron micrograph of a human coronary plaque showing loss of endothelial cells and a monolayer of platelets adhering to exposed subendothelial tissue.

denudation are those in which there is a heavy inflammatory component and many lipid-filled foam cells lying between fragmented collagen at the plaque surface. The thrombus which forms is stuck onto the luminal surface of the plaque which itself appears intact.

Deep plaque injury differs from the superficial injury in many ways. The basic process is one in which the plaque tears or splits open exposing its lipid core to blood in the lumen. The tear or split goes deep into the plaque and is of far greater magnitude than the loss of endothelium or superficial injury. The result of what is known as plaque tearing, fissuring, splitting or rupture is that blood enters the plaque from the lumen. Contact of blood with collagen and Tissue Factor (TF) leads to thrombus formation within the plaque, which is expanded and distortedinitially from within.

The type of plaque which undergoes this fissuring process is predominantly one that contains a large core of extracellular lipid, and it is into this core that the tear extends. ${ }^{18}$ Plaques in which the extracellular lipid core makes up more than $40 \%$ of the overall plaque volume are particularly susceptible. A few plaque tears occur in the plane between a plate of calcium and the adjacent intima and bear no relation to lipid pools. This type of tearing may be due to shear stresses within the plaque and may be more common in older subjects.

Plaque fissuring comes in various shapes and sizes. The tear may be small, measuring 100-200 $\mu \mathrm{m}$ across. Such tears allow blood to enter and expand the plaque but may not result in thrombus formation in the arterial lumen. Such fissures would lead to sudden increases in the degree of stenosis but not necessarily either clinical symptoms or angiographic alterations in the outline of the plaque. Subclinical episodes of plaque insta- $\mathbb{Q}$ bility, intraplaque thrombosis, and fissuring $\overrightarrow{\overrightarrow{0}}$ are a major factor in disease progression once 3 advanced plaques are present.

Larger plaque fissures and tears may be associated with a combination of intraplaque $\vec{\nabla}$ thrombosis and thrombosis within the lumen over the site of plaque disruption (fig 6). The thrombus over the site of disruption is made up of both fibrin and platelets whereas within the plaque itself platelets often predominate.

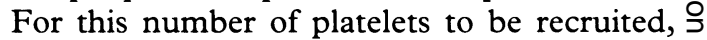
blood must enter and leave the interior of the $\frac{7}{2}$ plaque via the tear site for an appreciable time. The thrombus over the tear projects $N$ into the lumen but does not prevent antero- $N$ grade flow. This type of mural thrombus over N a fissured plaque produces the typical angio- $\sigma$ graphic appearances highlighted as being present in the "culprit" vessel in unstable angina $\frac{}{\Phi}$ and designated as type II. ${ }^{19}$ The ragged out- $\stackrel{\oplus}{?}$ line (which contrasts with the smooth stenosis 0 (type I) typical of stable angina) and overhanging edges are produced by contrast $\stackrel{\mathbb{\Omega}}{\stackrel{\circ}{\circ}}$ medium entering the tear and by thrombus $\stackrel{\otimes}{\AA}$ projecting into the lumen. Angioscopy can demonstrate both the mural thrombus and o the torn plaques. ${ }^{20}$ Thrombi projecting into응 the lumen but not preventing anterograde flow are associated with embolisation of platelet clumps into the distal vascular bed. Such platelet emboli are associated with focal microscopic myocardial necrosis. ${ }^{2122}$ 


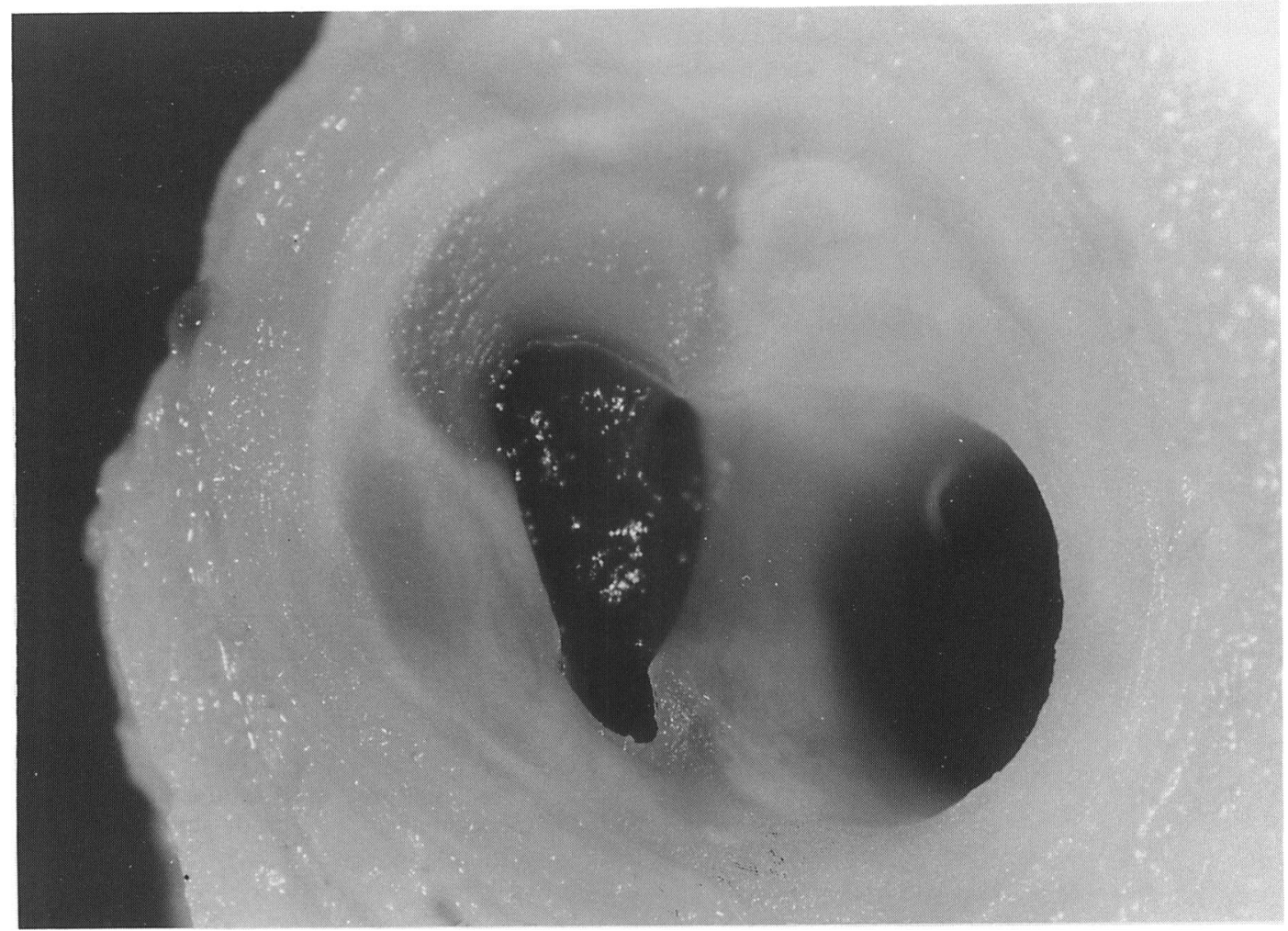

Figure 6 Thrombus projecting into the lumen of a human coronary artery from the centre of a ruptured plaque.

Lesions which progress to become occlusive enter a final phase of intraluminal thrombosis in which the thrombus is poor in platelets and rich in a loose network of fibrin that enmeshes red cells. This final occluding phase thrombus is very susceptible to fibrinolysis whether natural or iatrogenic. The structure of the final phase occluding thrombus, which is rich in fibrin and red cells, suggests that it forms within a very slow flowing or static column of blood.

It is likely that many variables determine whether an unstable plaque proceeds rapidly to an occlusive thrombus with the potential for acute infarction or persists at an intermediate stage as a mural non-occlusive thrombus. ${ }^{23}$ The factors which favour rapid progression to occlusion are probably enhanced thrombotic activity or diminished fibrinolytic activity, larger fissures with a greater thrombogenic potential, and severe reduction in flow mediated by local spasm or distal vascular shutdown.

Large fissures associated with occlusion and infarction are often very complex. The plaque may develop a spiral tear over several millimetres and an intimal flap may be raised. Some plaques develop a proximal entry point tear and the contents are extruded as a plug of cholesterol-rich debris via a distal break. The lumen in these cases may be occluded by a mixture of thrombus and debris distal to rather than at the plaque site itself. The major plaque tears probably cause thrombosis in which the vessel lumen cannot be reopened by lysis and reoccludes very rapidly.

Course of unstable plaques with fissuring The events which follow an episode of plaque fissuring have a dynamic course. The appearance found at necropsy is a reflection of the interaction of processes such as thrombus propagation, thrombolysis, organisation, and smooth muscle proliferation. The final result can range from restoration of a virtually normal lumen size to chronic total obstruction (fig 7).

The healing process after an episode of fissuring is mediated by what is the ubiquitous vascular repair response-smooth muscle proliferation and collagen production. The fissure site is thus resealed, incarcerating within the plaque a mass of thrombus which will itself stimulate further collagen production. In a sense the plaque is restabilised but it has also been stimulated into a growth phase.

If thrombus has formed within the lumen this is either removed by lysis or becomes invaded by capillaries and replaced by fine connective tissue in the process of organisation. The lumen may be chronically occluded or replaced by several vascular channels.

Very large plaque tears lead to the loss of the whole cap of the plaque with washout of the lipid contents, leaving a crater which partially fills with thrombus. In the carotid arteries and aorta such craters commonly become chronic and provide a source of distal platelet emboli. Such chronic craters are rare in the coronary arteries but may be outlined on angiography in some patients who have had fibrinolytic therapy for acute infarction.

Angiography based on the criteria of a ragged outline and overhanging edges does not detect all plaque fissures even when it is carried out at necropsy with high resolution film and lack of movement. Recanalised vessels with several lumens will reproduce exactly the 


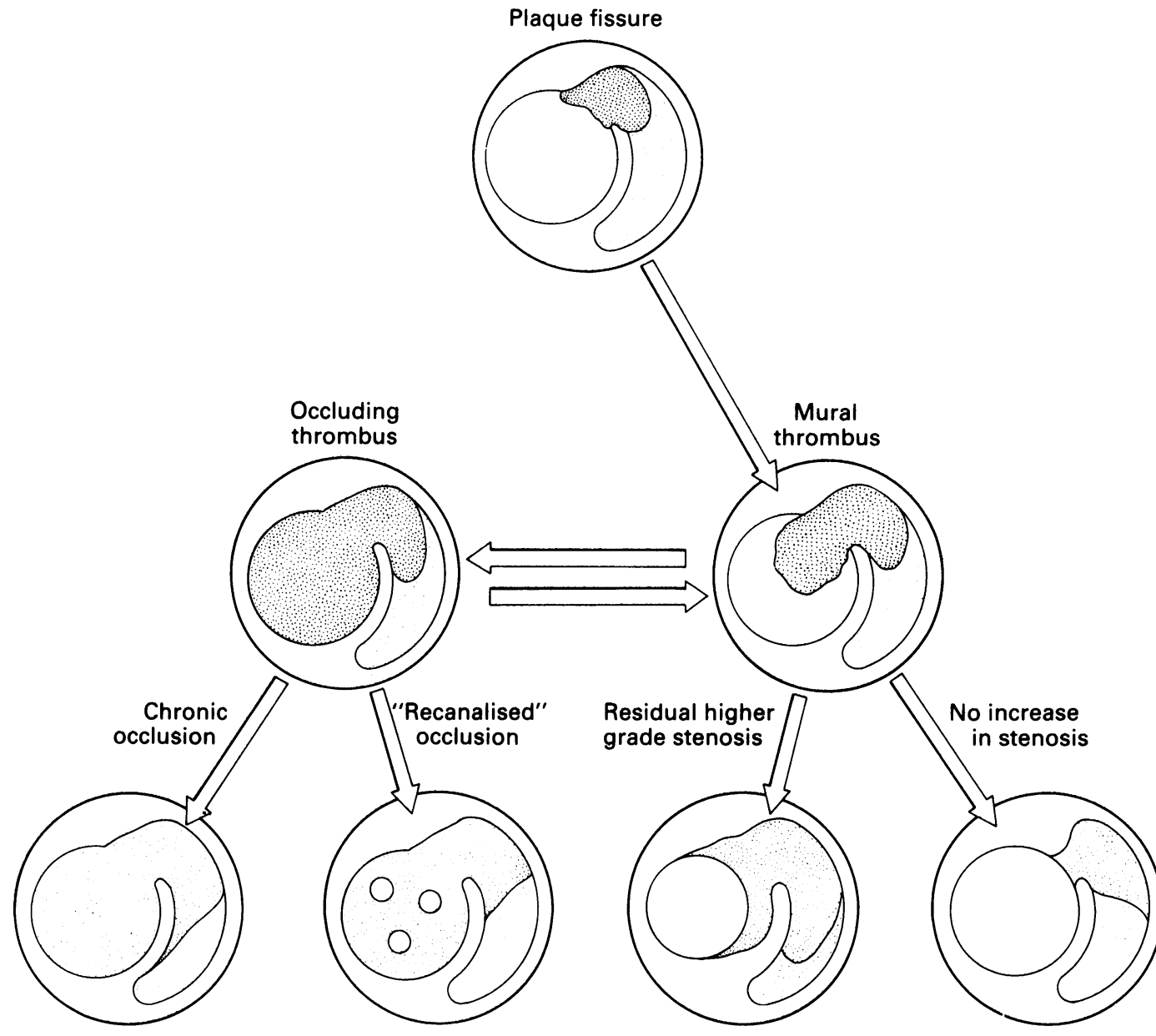

Figure 7 Potential outcomes of an episode of plaque fissuring.

irregular outlines of active fissures. Thus clinical angiography cannot be very sensitive or specific for plaque fissures. The best evidence that subclinical episodes of plaque fissuring occur comes from postmortem studies of control populations in which coronary atherosclerosis was present but was not the cause of death. ${ }^{24}$ At least one episode of recent fissuring but without intraluminal thrombus formation was found in $8 \%$ of individuals whereas in those with hypertension or diabetes the figure was $16 \%$. These facts show that fissuring is a common event in subjects with atherosclerosis and that it is an integral part of plaque progression whether or not clinical symptoms occur at the time. Numerous factors must determine whether the stimulus of a fissure produces significant intraluminal thrombus. Predominant among these factors are the size of the fissure and the balance of thrombotic/lytic systemic factors at the time. Some intraplaque thrombus is, however, inevitable. Drugs with an antithrombotic action might not only prevent the development of acute clinical episodes but also delay the progression of atherosclerosis by reducing plaque growth.

\section{Cause of plaque instability}

Given that plaque instability is the cause of the thrombosis which precipitates both silent episodic plaque growth and acute ischaemic syndromes, understanding its pathogenesis might lead to suggestions how it could be avoided.

Tearing could result either from reduced mechanical strength or from enhanced stress on the tissues. One solid fact to emerge from several pathological studies is that the plaque at risk has a large lipid core and that it is the plaque cap tissue that tears.

In one approach the alterations in the distribution of circumferential wall stress in sys- $\frac{7}{2}$ tole across this type of lipid-rich pool have been simulated. ${ }^{18}$ Stress on the cap is much of increased because the soft lipid core cannot $\tilde{O}$ carry a load, which has to be redistributed. N Stiff cap tissue and an eccentric position of the plaque enhance this tendency for stress to 0 be concentrated on the cap at its junction with the more normal vessel wall.

In other approaches the cap tissue of aortic plaques was mechanically tested, analysed biochemically, and the proportions of cell types measured. ${ }^{25}$ The caps of plaques which $\Omega$ had ulcerated had a reduced mechanical 2 strength, reduced collagen content, a reduced $\delta$ smooth muscle content, and an increased proportion of lipid-filled macrophages.

Plaque instability is thus seen to be a function of increasing extracellular lipid content, increasing macrophage content, and diminishing smooth muscle content. The decline in 
smooth muscle numbers may indicate that the normal reparative process which maintains plaque stability has been overcome.

\section{Regression of atherosclerosis}

Animal models suggest that regression of lesions can occur after plasma lipid concentrations are reduced. What does regression mean in the context of animal models? Many use the simple estimation of the proportion of the intimal surface occupied by plaques or by stainable lipid. What is required in humans is a reduction in plaque mass leading to angiographic improvement. Given that many high grade stenoses in humans consist largely of collagen this may not be readily achieved. Many patients with symptomatic atherosclerosis would happily settle for a guarantee of no further acute episodes or progression of their stenoses. Animal models in which the qualitative changes in the constituents of plaques were examined after lipid lowering showed a reduction in lipid with a concomitant increase in smooth muscle cells and collagen. ${ }^{26}$ These are just the changes that would be associated with an increase in plaque stability. If the same phenomenon occurs in humans an increase in plaque stability would reduce subsequent acute clinical events and the rate of angiographic progression.

1 Ross R. The pathogenesis of atherosclerosis-an update. N Engl ₹ Med 1986;314:488-500.

2 Steinberg D, Parthasarathy S, Carew T, Khoo J, Witztum J. Beyond cholesterol. Modifications of low-density lipoprotein that increases its atherogenicity. $N$ Engl $f$ Med 1989;320:915-24.

3 Stary $\mathrm{H}$. Evolution and progression of atherosclerotic lesions in coronary arteries of children and young adults. Arteriosclerosis 1989;9:1-19.

4 Restrepo C, Tracy R. Variations in human aortic fatty streaks among geographic locations. Atherosclerosis 1975; 21:179-93.

5 Deupree $R$, Fields $R$, McMahon C, Strong J. Atherosclerotic lesions and coronary heart disease. Key relationships in necropsied cases. Lab Invest 1973;28. 252-62.

6 Robertson W, Strong J. Atherosclerosis in persons with hypertension and diabetes mellitus. Lab Invest 1968 18:538-51.

7 PDAY Research Group. Relationship of atherosclerosis in young men to serum lipoprotein cholesterol concentrations and smoking. A preliminary report from the pathological determinant of atherosclerosis in youth study. FAMA 1990;264:3018-24.

8 Glagov S, Weisenberd E, Zarins C, Stankunavicius R Kolettis G. Compensatory enlargement of human atherosclerotic coronary arteries. N Engl f Med 1987; 316:1371-5.

9 Faggiotto A, Ross R, Harker L. Studies of hypercholesterolaemia in the non-human primate. I Changes that lead to fatty streak formation. Arteriosclerosis 1984, 4:323-40.

10 Faggiotto A, Ross R. Studies of hypercholesteolaemia in non-human primates. II Fatty streak conversion to fibrous plaque. Arteriosclerosis 1984;4:341-56.

11 de Feyter $P$, Serruys $P$, Davies $M$, Richardson $P$, Lubsen $J$, Oliver $M$. Quantitative coronary angiography to measure progression and regression of coronary atherosclerosis. Value, limitations, and implications for clinical trials. Circulation 1991;84:412-23.

12 Hangartner J, Charleston A, Davies M, Thomas A Morphological characteristics of clinically significan coronary artery stenosis in stable angina. Br Heart 1986;56:501-8.

13 Saner H, Gobel F, Salomonowitz E, Erlien D, Edwards J. The disease free wall in coronary atherosclerosis: its relation to degree of obstruction. $\mathcal{F} \mathrm{Am}$ Coll Cardiol 1985;6:1096-9.

14 Davies M, Woolf N, Rowles P, Pepper J. Morphology of the endothelium over atherosclerotic plaques in human the endothelium over atherosclerotic plaques

15 Burrig $K$. The endothelium of advanced arteriosclerotic plaques in humans. Arterioscl Thromb 1991;11:1678-89.

16 Bitker H, Ravkilde J, Sigaard P, Jirgensen P, Hirder M, Thygesen K. Gradation of unstable angina based on sensitive immunoassay for serum creatine kinase $M B$. Br Heart f 1991;65:72-6.

17 Davies M. A macroscopic and microscopic view of coronary thrombi. Circulation 1990;82:1138-46.

18 Richardson $\mathrm{P}$, Davies $\mathrm{M}$, Born $\mathrm{G}$. Influence of plaque configuration and stress distribution on fissuring of coronary atherosclerotic plaques. Lancet 1989;ii:941-4.

19 Ambrose J, Winters S, Arora $\mathrm{R}$, et al. Coronary angiograph morphology in acute myocardial infarction: link between the pathogenesis of unstable angina and myocardial infarction. F Am Coll Cardiol 1985;6:1233-8.

20 Forrester J, Litvak F, Grundfest W, Hickey A. A perspective of coronary disease seen through the arteries of a living man. Circulation 1987;75:505-13.

21 Falk E. Unstable angina with fatal outcome: dynamic coronary thrombosis leading to infarction and/or sudden death. Circulation 1985:71:699-708.

22 Davies $M$, Thomas A, Knapman $P$, Hangartner $R$ Intramyocardial platelet aggregation in patients with Intramyocardial platelet aggregation in patients with unstable angina suffering sudden

23 Fuster V, Badimon L, Badimon J, Chesebro J. The pathogenesis of coronary artery disease and the acute co nary syndrome II. $N$ Engl ₹ Med 1992;326:310-8.

24 Davies M, Bland J, Hangartner J, Angelini A, Thomas A Factors influencing the presence or absence of acute coronary artery thrombi in sudden ischaemic death. Eur Heart $\mathcal{f} 1989 ; 10: 203-8$.

25 Lendon C, Davies M, Born G, Richardson P. Atherosclerotic plaque caps are locally weakened when macrophages density is increased. Atherosclerosis 1991;87: phages

26 Small D, Bond $M$, Waugh D, Prack M, Sawyer J. Physicochemical and histological changes in the arterial wall of nonhuman primates during progression and regression of atherosclerosis. I Clin Invest 1984;73 1590-605. 